\title{
Tunneling dissociation from a double well via path integrals
}

\author{
Theodosios G. Douvropoulos ${ }^{\text {a) }}$ \\ Physics Department, National Technical University, Athens, Greece
}

Cleanthes A. Nicolaides ${ }^{\text {b) }}$

Physics Department, National Technical University, Athens, Greece, and Theoretical and Physical

Chemistry Institute, National Hellenic Research Foundation, 48 Vasileos Constantinou Avenue, Athens 11635 Greece

(Received 12 February 2003; accepted 31 July 2003)

\begin{abstract}
It is shown how the semiclassical theory of path integrals can be implemented in a practical manner for the analysis of a potential that combines the two-state system of a double well potential (DWP) with decay into a continuous spectrum. This potential may correspond to a variety of physical situations in physics and chemistry. The structure of the formalism and of the results is such that it allows computation not only for analytic but also for numerically given potentials. The central theme is the determination of the energy-dependent Green's function, which is shown to consist of a regular part and a part containing simple and double complex poles. These poles represent the position of the energy levels, as well as the energy widths and shifts due to the interaction with the continuous spectrum. When applied to the bound DWP without tunneling, the theory is shown to reduce in certain limits to known results from the Jeffreys-Wentzel-Kiamers-Bhrillouin approximation. If the system is taken to be prepared in the first well, the interactions with the remaining of the potential lead to two types of transition rates. One represents the transient motion toward a virtual equilibrium state of the DWP. It emerges as a positive imaginary part of the self-energy. The other represents the decay into the continuum and emerges as a negative imaginary part of the pole. Comparison of the two mechanisms of nonstationarity is made for different magnitudes of the second barrier relative to the first one. Since the system decays to the continuum while oscillating, the theory obtains a correction to the frequency of oscillation in the DWP due to the interaction with the continuum. This phenomenon is observable in real two-state systems, if an external perturbation which affects mainly one state converts it into a resonance state. (C) 2003 American Institute of Physics. [DOI: 10.1063/1.1612482]
\end{abstract}

\section{INTRODUCTION}

Among the prototypical systems for the demonstration of the physics of the phenomenon of tunneling, are the single well potential (SWP) with an unbound region and the bounded double well potential (DWP). Aspects of the physics of these two systems have been published in numerous publications, using analytic potentials and/or the standard model of the semiclassical version of quantum mechanics, namely the Jeffreys-Wentzel-Kramers-Brillouin (JWKB) approximation. ${ }^{1,2}$

The work reported here has a dual purpose: First, to seek knowledge about the physics of a one-dimensional (1D) potential whose generic form is as in Fig. 1, using semiclassical physics. Figure 1 constitutes the semiclassical representation of the coupling of two discrete spectra, of which one is coupled to a continuous spectrum. The continuous spectrum has a lower bound if it corresponds to a system without an external field. Second, to do so by applying the formalism of path integrals and Green's functions, thereby providing additional information as to the capacity of this type of methodology in the treatment of unstable systems of coupled states.

In the potential of interest, one expects oscillatory, se-

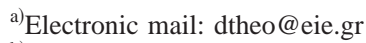

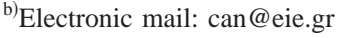

quential and dissipative tunneling, i.e., dynamics that may characterize a number of situations in physics and chemistry, regarding, e.g., atomic and molecular spectra, various molecular rearrangements, and one-dimensional solid state structures along a reaction coordinate leading to dissociation, quantum transport in the solid state and in optical lattices, nuclear and elementary particle resonances, and field theory. For example, a specific model of this figure, using square wells, has been invoked by Faist et al. ${ }^{3}$ for the analysis of control, by tunneling, of interference in optical absorption in quantum wells. A quantum mechanical version of the interactions and processes described by Fig. 1 was examined for coupled states of a real two-electron system via $a b$ initio calculation in Ref. 4. Specifically, two neighboring doubly excited states in the continuous spectrum are coupled by an electric dipole field. One of them is discrete, of even parity, $\left(2 p^{23} P\right)$, and the other one is unstable, of odd parity, $\left(2 s 2 p^{3} P^{0}\right)$, decaying into the $1 s \epsilon p^{3} P^{0}$ continuum of scattering states. The lifetimes of the two states differ by orders of magnitude, one of the discrete state being of the order of $10^{-9} \mathrm{~s}$ (radiative decay) and one of the resonance state being of the order of $10^{-13} \mathrm{~s}$. First principles theory and computation demonstrated quantitatively that when the two states are coupled by the electric field the discrete state acquires a finite width due to autoionization, and its lifetime is reduced. ${ }^{4}$ 


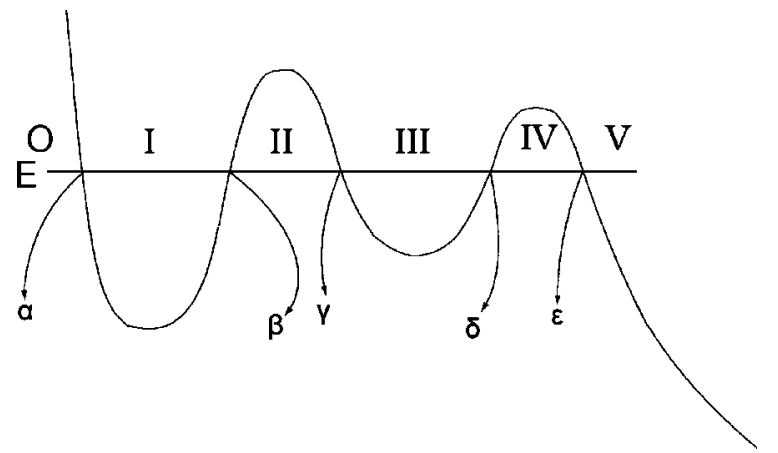

FIG. 1. The double-well potential plus tunneling into the continuum, examined in this work.

Similar situations are possible in molecular spectra that exhibit a variety of interactions and crossings or near-crossings of levels.

As regards the dissipative tunneling through a single finite barrier, its extensive analysis in the literature has emphasized the derivation and calculation of time-independent quantities, such as rates and transmission coefficients. For the SWP, an expression for the energy shift caused by the interaction with the continuum has also been derived. ${ }^{5}$

As regards the bound system of the DWP, the physics of tunneling that has attracted attention as well as application is normally analyzed in terms of the energy separation of the doublets (for initially degenerate states of each well) and of the related period of oscillation. For discussions and results of semiclassical treatments of this problem, the reader is referred to the books of Landau and Lifshitz ${ }^{1}$ and of Merzbacher, ${ }^{2}$ to the review on "Quantum Dynamics in LowTemperature Chemistry" by Benderskii, Goldanskii, and Makarov, ${ }^{6}$ and to the papers of Miller ${ }^{7}$ on symmetric and asymmetric DWP and of Holstein ${ }^{8,9}$ on symmetric DWP.

The present work (see also Ref. 5) implements a semiclassical path-integral approach and emphasizes the calculation of energy-dependent Green's functions [rather than the Hermitian $\left.g(E) \equiv \operatorname{trace}(E-H)^{-1}\right]$, and use of their truncated Fourier transform (from $E=0$ to $E=\infty$ rather than from $E=-\infty$ to $E=+\infty$ ), for the calculation of the timedependent propagator $G_{>}(t), t>0$. Discussions on the foundations and methodology of the semiclassical approach as regards the calculation of propagation amplitudes and of phase changes at the turning points of allowed and forbidden regions, can be found in the book of Feynman and Hibbs, ${ }^{10}$ in the reviews of Benderskii et al. ${ }^{6}$ and of Berry and Mount, ${ }^{11}$ and in the papers of Gutzwiller, ${ }^{12}$ McLaughlin, ${ }^{13}$ Miller, ${ }^{7,14}$ Holstein and Swift, ${ }^{15}$ and of Holstein. ${ }^{8,9,16}$

It is worth pointing out that when the potential of Fig. 1 is inverted, as is done with simpler potentials in the "instanton" model, e.g., Refs. 6, 9, 17-21, the topology of allowed and forbidden regions remains the same, contrary to the case of the DWP.

The main goal of the problem which is treated in this paper is to derive and interpret expressions in terms of a semiclassical Green's function, $G_{s c}(E)$, corresponding to the motion of a wave packet which is initially localized in the left well of Fig. 1 and which propagates according to this potential. $G_{s c}(E)$ consists of two major terms. One is regular and one has poles, the latter being associated with the interesting features of the dynamics. The results are general and can be applied to any analytic or numerical potential having this form. In the course of the solution of this problem, the DWP is also examined anew, and results beyond the JWKB approximation are obtained.

\section{THEORY AND CALCULATION OF THE SEMICLASSICAL GREEN'S FUNCTION, $G_{s c}(E)$}

The aim of the herein implemented path-integral formalism is the calculation of $G_{s c}(E)$ and its poles for the potential of Fig. 1. In total there are six regions for motion. The classically allowed are denoted by I, III, and V. The classically forbidden are the O, II, and IV. The DWP corresponds to the regions $\mathrm{O}$, I, II, III, with the second barrier being replaced by an infinite wall. Because of the complexity of the problem, it is necessary to define and utilize a number of quantities, and this makes the paper rather long. The solution has employed a building-up methodology, starting from the simpler parts and adding possibilities until the complete problem is solved.

The calculation could in principle be done by taking the Fourier transform of the classical limit of Feynman's timedependent propagator between points $r_{1}$ and $r_{2}, G_{s c}(t)$, where, in general,

$$
\begin{aligned}
G_{s c}(t) & \equiv G_{s c}\left(r_{1}, t ; r_{2}, 0\right) \\
& =\frac{1}{\sqrt{2 \pi i \hbar}} \sum_{\text {paths } j} \sqrt{D} \exp \left[\frac{i}{\hbar} S_{c l}^{j}-\frac{i}{2} \mu \pi\right] .
\end{aligned}
$$

$D$ is the Van Vleck determinant, $S_{c l}^{j}$ is the classical action along the path $j$, and $\mu$ is the Maslov index. For the present problem points $r_{1}$ and $r_{2}$ belong to region $\mathrm{I}$.

The acknowledged great difficulty in computing $G_{s c}(t)$, is obviated (relatively speaking) by focusing on calculating $G_{s c}(E)$ directly. In this case, the existence of classically forbidden regions requires the possibility of some type of analytic continuation through such regions, which is formally achieved by complexifying time or dynamical variables. ${ }^{1,9,11-19}$ Once the solution to this problem is understood, the calculation of $G_{s c}(E)$ requires a meticulous accounting of all possible paths and a good understanding of the related combinatorics. In so doing, the question of the proper Maslov indices, i.e., of the phase change at the turning points before a forbidden or an allowed region is entered, is of crucial importance. It is in this respect that the papers of Holstein ${ }^{8,9,15,16}$ have been enlightening to us, since his analysis and the set of rules listed by him provide the framework for the formulation of systematic procedures toward the calculation of $G_{s c}(E)$ in the case of tunneling, ${ }^{5}$ and this work.

Once $G_{s c}(E)$ is known, one can obtain, via Fourier transform, the time-dependent propagator, $G_{>}(t)$, for $t>0$, and from there the survival probability of the initial state, $P(t)^{5}$

The path integral approach requires the determination of the amplitudes for motion for all possible paths from $r_{1}$ to $r_{2}$, where both $r_{1}$ and $r_{2}$ are in region I. In developing and 
applying the corresponding methodology, the resulting complexity requires extensive use of symbolism. We follow and extend the one given in Ref. 5.

The strategy that we adopted for dealing with this problem is to define the various regions (Fig. 1) and to account progressively for their contribution. It has already been shown that propagation in region $\mathrm{O}$ does not contribute to $G_{s c}(E) .^{5}$ Therefore, we considered four categories: (i) Propagation in region I only; (ii) Propagation in regions I and II only; (iii) Propagation in regions I, II, and III only; (iv) Propagation in regions I, II, III, and IV.

A considerable amount of "algebra" is needed for deriving the amplitudes. For reasons of economy, below we only give the results for each category and the description of the symbolism.

(i) The amplitude is simply

$$
J_{1}=A_{r_{1} r_{2}}^{\mathrm{I}} \text {. }
$$

The symbol $A_{r_{1} r_{2}}^{\mathrm{I}}$ is the amplitude for propagation from point $r_{1}$ to point $r_{2}$ in all possible ways (paths), with the restriction that the particle remains in region I during the propagation.

$$
\text { (ii) } J_{2}=\left(\mathrm{I}-\mathrm{II}^{*}\right)^{r_{1} / \beta} A_{\beta r_{2}}^{\mathrm{I}} \text {. }
$$

The symbol in parenthesis means the following: The Roman numerals represent the regions that are covered during the propagation, in all possible ways and at least once for each propagation. The region with the asterisk is the last one that is met in the propagation. The two upper indices express, in order of their appearance, the initial and the final point.

Expression (3) has the following meaning: Since the final point of the propagation is point $r_{2}$, if we want to interchange regions I and II during the propagation (in all possible ways), we must finally come to point $\beta$ which is the boundary between these two regions. The quantity (I-II*) $)^{r_{1} / \beta}$ describes the above procedure. When point $\beta$ is reached, we account for all possible paths which lead to point $r_{2}$ while remaining in region I. The last procedure is described by the quantity $A_{\beta r_{2}}^{\mathrm{I}}$. Region I cannot be entered during the last part of the propagation, because this has already been taken in account through the quantity (I-II* $)^{r_{1} / \beta}$.

(iii)

$$
\begin{aligned}
J_{3}= & \left.\left.(\mathrm{I}-\mathrm{II})^{*}\right)^{r_{1} / \gamma}(\mathrm{III}-\mathrm{II})^{*}\right)^{\gamma / \beta} X\{\mathrm{I}, \mathrm{II}, \mathrm{III} ; \beta, \gamma\}\left[\left(\mathrm{I}^{*}-\mathrm{II}\right)^{\beta / r_{2}}\right. \\
& \left.+A_{\beta r_{2}}^{\mathrm{I}}\right],
\end{aligned}
$$

where the quantity $X\{\mathrm{I}, \mathrm{II}, \mathrm{III} ; \beta, \gamma\}$ is defined as:

$$
\begin{aligned}
& \left.X\{\mathrm{I}, \mathrm{II}, \mathrm{III} ; \beta, \gamma\}=1+\left(\mathrm{I}-\mathrm{II}{ }^{*}\right)^{\beta / \gamma}(\mathrm{III}-\mathrm{II})^{*}\right)^{\gamma / \beta} \\
& \left.+\left(\left(\mathrm{I}-\mathrm{II}{ }^{*}\right)^{\beta / \gamma}(\mathrm{III}-\mathrm{II})^{*}\right)^{\gamma / \beta}\right)^{2}+\ldots \\
& =\frac{1}{\left.1-(\mathrm{I}-\mathrm{II})^{*}\right)^{\beta / \gamma}\left(\mathrm{III}-\mathrm{I}^{*}\right)^{\gamma / \beta}} .
\end{aligned}
$$

Here, things are more complicated. Given two points, Eq. (4) describes propagation consisting of two parts. In the first part, we interchange three regions, I, II, and III, in all possible ways. Again, eventually we must arrive at point $\beta$. The first quantity, $\left(\mathrm{I}-\mathrm{II}^{*}\right)^{r_{1} / \gamma}$ (III-II*) $)^{\gamma / \beta}$, describes one such propagation, starting from point $r_{1}$ and not traversing region I again, after region III is reached. But, it is possible to traverse region I again (while interchanging with region II) and then interchange regions III and II, and so on. Hence, it can be understood that we can construct a polynomial of the variable $\left.(\mathrm{I}-\mathrm{II})^{*}\right)^{\beta / \gamma}(\mathrm{III}-\mathrm{II} *)^{\gamma / \beta}$, which describes the interchange of the three regions, starting and terminating at point $\beta$, and which is of infinite order since there is an infinity of paths corresponding to this procedure. The amplitude for such propagation is represented by the quantity $X\{\mathrm{I}, \mathrm{II}, \mathrm{III} ; \boldsymbol{\beta}, \boldsymbol{\gamma}\}$.

Finally, after reaching point $\beta$ for the last time during this interchange, we must propagate to point $r_{1}$ without passing through region III again. This last part of the propagation is described by the quantity (I*-II) ${ }^{\beta / r_{2}}+A_{\beta r_{2}}^{\mathrm{I}}$, which includes the case where the particle propagates only in region I $\left(A_{\beta r_{2}}^{\mathrm{I}}\right)$.

(iv) The amplitude of the fourth category involves all four regions in all possible ways. We realize that this implies consideration of three regions at a time, without jumps. The possible triplets are: (I-II-III) and (II-III-IV), and we must consider all possible interchanges. For example, the symbol $\left(\mathrm{I}-\mathrm{II}-\mathrm{III}{ }^{*}\right)^{\beta / \delta}$ means all possible interchanges having as first the region I and last the region III, while propagating from point $\beta$ to point $\delta$. We remind ourselves that every possible path must engage all three regions. So we have:

$$
\begin{aligned}
J_{4}= & \left.(\mathrm{I}-\mathrm{II}-\mathrm{III})^{*}\right)^{r_{1} / \delta}\left(\mathrm{IV}-\mathrm{III}-\mathrm{II}{ }^{*}\right)^{\delta / \beta} Y\{\mathrm{I}, \mathrm{II}, \mathrm{III}, \mathrm{IV} ; \beta, \delta\} \\
& \times Z\left\{\mathrm{I}, \mathrm{II}, \mathrm{III} ; \beta, \gamma, r_{2}\right\},
\end{aligned}
$$

where

$$
\begin{aligned}
Y\{\mathrm{I}, \mathrm{II}, \mathrm{III}, \mathrm{IV} ; \beta, \delta\}= & \left.1+(\mathrm{I}-\mathrm{II}-\mathrm{III})^{*}\right)^{\beta / \delta}(\mathrm{IV}-\mathrm{III}-\mathrm{II} *)^{\delta / \beta} \\
& \left.+\left((\mathrm{I}-\mathrm{II}-\mathrm{III})^{*}\right)^{\beta / \delta}\left(\mathrm{IV}^{\mathrm{III}-\mathrm{II}}\right)^{\delta / \beta}\right)^{2}+\ldots \\
= & \frac{1}{\left.1-(\mathrm{I}-\mathrm{II}-\mathrm{III})^{*}\right)^{\beta / \delta}\left(\mathrm{IV}-\mathrm{III}-\mathrm{II}^{*}\right)^{\delta / \beta}} .
\end{aligned}
$$

The quantity $Y\{\mathrm{I}, \mathrm{II}, \mathrm{III}, \mathrm{IV} ; \beta, \delta\}$ is a polynomial of the variable $(\text { I-II-III*) })^{\beta / \delta}(\text { IV-III-II*) })^{\delta / \beta}$ of infinite order. This variable describes the interchange of regions I, II, and III, starting at point $\beta$ and terminating at point $\delta$, (in all possible ways) followed by the interchange of regions IV, III, and II, starting at point $\delta$ and terminating at point $\beta$ (in all possible ways).

The quantity $Z\left\{\mathrm{I}, \mathrm{II}, \mathrm{III} ; \beta, \gamma, r_{2}\right\}$ consists of: (A) Direct propagation to the point $r_{2}$ through region I; (B) Exhaustion of the pair (I-II) and propagation to $r_{2}$; (C) Exhaustion of the triplet (I-II-III) and then, either use of the pair (I-II) and propagation, or direct propagation. Therefore, the final result is:

$$
\begin{aligned}
Z\left\{\mathrm{I}, \mathrm{II}, \mathrm{III} ; \beta, \gamma, r_{2}\right\}= & \left((\mathrm{I}-\mathrm{II} *-\mathrm{III})^{\beta / \beta}+1\right) \\
& \times A_{\beta r_{2}}^{\mathrm{I}}\left(1+\left(\mathrm{I}^{-\mathrm{II}}\right)^{\beta / \beta}\right) .
\end{aligned}
$$

As explained in Refs. 8, 15, the semiclassical propagator for motion from point $r_{1}$ to point $r_{2}$ of region I is the sum of the above four amplitudes, multiplied by the factor $1 /\left[2 \pi \sqrt{k\left(r_{1}\right) k\left(r_{2}\right)}\right]$, where $k(r)$ is the momentum, defined by Eq. (6) below. 
We now have to compute all the terms appearing in the above relations. These terms consist of triplets for propagation involving three regions, or of pairs for propagation involving two regions, or, in the simplest case, of single amplitudes for propagation in only one region. In what follows, for reasons of economy we present only the main results, without the intermediate steps of analytic computation.

We begin by defining certain quantities. These are divided into two categories: $(\alpha)$ Well factors [Eqs. (6)-(10b)], and $(\beta)$ Barrier factors [Eqs. (11a) $-(13)]$ :

WELL FACTORS:

$$
\begin{aligned}
& k(r)=\{2[E-V(r)]\}^{1 / 2}, \\
& \lambda(r)=\int^{r} k(x) d x, \\
& \mu(r)=\lambda(r)-\theta, \\
& \theta=\lambda(\alpha)-\frac{\pi}{4}, \\
& \xi(r)=\lambda(r)-\varphi, \\
& \varphi=\lambda(\gamma)-\frac{\pi}{4}, \\
& \exp i[2 \mu(\beta)] \equiv \beta^{x}, \\
& \exp i[2 \xi(\delta)] \equiv \delta^{x} .
\end{aligned}
$$

\section{BARRIER FACTORS:}

$$
\begin{aligned}
& \kappa_{1}=\sqrt{2} \int_{\beta}^{\gamma} d x \sqrt{V(x)-E}, \\
& \kappa_{2}=\sqrt{2} \int_{\delta}^{\varepsilon} d x \sqrt{V(x)-E}, \\
& \frac{\exp \left[-2 \kappa_{1}\right]}{1+\frac{1}{4} \exp \left[-2 \kappa_{1}\right]}=x, \\
& \frac{\exp \left[-2 \kappa_{2}\right]}{1+\frac{1}{4} \exp \left[-2 \kappa_{2}\right]}=y .
\end{aligned}
$$

We are now ready to obtain $G_{s c}(E)$.

$$
\text { (i) } J_{1}=A_{r_{1} r_{2}}^{\mathrm{I}}
$$

and, after some algebra,:

$$
\begin{aligned}
J_{1}= & -2 i \sin \mu\left(r_{1}\right) \exp \left[-i \mu\left(r_{2}\right)\right] \\
& +\frac{4 \sin \mu\left(r_{1}\right) \sin \mu\left(r_{2}\right)}{1-i \beta^{x}} ; \\
\text { (ii) } \quad J_{2}=(\mathrm{I}-\mathrm{II})^{r_{1} / \beta} A_{\beta r_{2}}^{\mathrm{I}}, & \\
J_{2}= & -\frac{4 \sin \mu\left(r_{1}\right) \sin \mu\left(r_{2}\right)}{\left(1-i \beta^{x}\right)}+\frac{8 \sin \mu\left(r_{1}\right) \sin \mu\left(r_{2}\right)}{2\left(1-i \beta^{x}\right)+i x \beta^{x}} ;
\end{aligned}
$$

where

$$
\zeta=\frac{8 x^{2} \exp \left(2 \kappa_{1}\right) y \beta^{x} \delta^{x}\left(1-i \delta^{x}\right)}{\left[2\left(1-i \beta^{x}\right)+i x \beta^{x}\right]\left[2\left(1 i \delta^{x}\right)+i x \delta^{x}\right]-i x y \delta^{x}} .
$$

Summing the four amplitudes gives the sought after Green's function in an elegant form of two parts, the latter clearly exhibiting poles:

$$
\begin{aligned}
G_{s c}(E) & =\frac{1}{2 \pi \sqrt{k\left(r_{1}\right) k\left(r_{2}\right)}}\left(J_{1}+J_{2}+J_{3}+J_{4}\right) \\
& =\frac{1}{2 \pi \sqrt{k\left(r_{1}\right) k\left(r_{2}\right)}}\left\{-2 i \sin \mu\left(r_{1}\right) \exp \left[-i \mu\left(r_{2}\right)\right]+\frac{8 \sin \mu\left(r_{1}\right) \sin \mu\left(r_{2}\right)\left[2\left(1-i \delta^{x}\right)+i x \delta^{x}\right]}{\left[2\left(1-i \beta^{x}\right)+i x \beta^{x}\right]\left[2\left(1-i \delta^{x}\right)+i x \delta^{x}\right]-4 x^{2} \exp \left(2 \kappa_{1}\right) \beta^{x} \delta^{x}+\zeta}\right\} .
\end{aligned}
$$




\section{COMMENTS ON THE STRUCTURE OF $G_{s c}(E)$}

If the potential of Fig. 1 is known numerically, either from a quantum mechanical calculation or quasiempirically, then $G_{s c}(E)$ can be computed from first principles. In any case, we can extract the significant aspects of the physics directly from the form of Eqs. (14b), (15b), (16b), (17b), (17c), (18). These are connected to the fractional quantities where poles appear. The following comments are pertinent:

(1) Considering the forms of $J_{1}, J_{2}, J_{3}$, and $J_{4}$, and the way they were derived, we observe that each contribution has one pole term that cancels identically with a pole term of the previous contribution and one pole term that cancels identically with one of the next contribution. For example, the first term of Eq. (16b) describing $J_{3}$ is cancelled by the second term of Eq. (15b) describing $J_{2}$, while the second term of $J_{3}$ is canceled by the first term of Eq. (17b) describing $J_{4}$. This symmetric relationship is general and can be written as

$J_{n}=-J_{n+1}-J_{n-1}+F_{n}, n \geqslant 2$,

where $F_{n}$ is a function. This expresses the fact that the appearance of poles in the Green's function must represent the dependence of the propagation on the full potential and not on groups of locally coupled regions.

(2) The bound state problem is expressed by the factor ( 1 $-i \beta^{x}$ ) of Eq. (14b) (see next section). As soon as coupling through the barriers is allowed, this term is cancelled by $J_{2}$. It appears only together with other terms, representing the coupling of the bound problem to the tunneling process. For example, the term $i x \beta^{x}$ represents the coupling of the bound state $\left(\beta^{x}\right)$ with the first barrier $(x)$.

(3) Due to the existence of the two wells, one must expect the appearance of a product of two poles, provided the system is prepared in one well. Indeed, such a product is present in Eqs. (16b), (17b), where the term $4 x^{2} \exp \left(2 \kappa_{1}\right) \beta^{x} \delta^{x}$ represents the coupling of the two resonance states $\left(\beta^{x} \delta^{x}\right)$, which takes place again through a tunneling process $\left[x^{2} \exp \left(2 \kappa_{1}\right)\right]$.

(4) If the system is prepared in a stationary state of the bound DWP, a different type of pole must represent this situation, namely a double pole. This is seen as the product of the first two terms of the denominator of Eq. 18. It is clear that for such a pole to arise, we must have traversed at least the first three regions of interest. Indeed, we will show that the contributions from $J_{3}$ and $J_{4}$ produce such poles, and so connection to ammonia-type systems, where $\beta^{x}=\delta^{x}$, as well as to the asymmetric DWP can be made.

\section{POLES OF EACH CATEGORY $\boldsymbol{J}_{\boldsymbol{i}}$}

Apart from the derivation of the Green's function [Eq. (18)], it is significant to know the its poles, or the poles of parts of it. Because the potential contains classically forbidden regions, the poles turn complex as soon as tunneling is allowed. It is therefore expected that the presence of these regions introduces certain time scales, via the real and the imaginary parts of the poles. For example, the energy splitting that emerges from the real part of the double pole is connected to the frequency of oscillation, whereas the imaginary part of a pole is connected, in general, with a rate of time evolution. In other words, the nature of the poles determines the dynamics. In this section, the focus is on the poles of the Green's function, as they emerge from each amplitude. This is done by expanding the corresponding fraction around the energies of the unperturbed bound state problem. In this way, the poles depend directly on the initial preparation of the system. In order to compare with known results at the JWKB level, we apply our results to the harmonic oscillator well (HOW).

\section{$\left(J_{1}\right)$}

The pole is due to the denominator $1-i \beta^{x}$. Putting 1 $-i \beta^{x}=0$, results in $\exp [2 i \mu(\beta)]=-i$, and so

$$
2 \mu(\beta)=2 n \pi+3 \pi / 2 \text {. }
$$

\section{A. Application to the harmonic oscillator well}

From Ref. 5 we know that if the well is that of the harmonic oscillator then $\mu(\beta)=(E / \omega) \pi+(\pi / 4)$. It follows that Eq. (20) is equivalent to

$$
E_{n}=\left(n+\frac{1}{2}\right) \omega \text {. }
$$

Therefore, $J_{1}$ represents the exact bound state problem.

$\left(J_{2}\right)$

There is an additional pole, due to the denominator $\left\{2\left(1-i \beta^{x}\right)+i x \beta^{x}\right\}$. This term cannot become zero exactly. However, we may assume that, for realistic problems, $x \ll 1$ and therefore we can develop the denominator around the poles resulting from such an approximation: ${ }^{5}$

$$
\begin{aligned}
2(1- & \left.i \beta^{x}\right)+i x \beta^{x} \\
\simeq & {\left[2\left(1-i \beta^{x}\right)+i x \beta^{x}\right]_{E_{n}}+\left(\frac{d}{d E}\left[2\left(1-i \beta^{x}\right)+i x \beta^{x}\right]\right)_{E_{n}} } \\
& \times\left(E-E_{n}\right) .
\end{aligned}
$$

Carrying out the algebra for the case of the HOW leads to:

$$
\frac{1}{2\left(1-i \beta^{x}\right)+i x \beta^{x}} \simeq \frac{\omega}{2 \pi(f(x)+i d(x))}{ }_{E-\left(E_{n}-\frac{\omega}{2 \pi} \frac{x f(x)}{f(x)^{2}+d(x)^{2}}-i \frac{\omega}{2 \pi} \frac{x d(x)}{f(x)^{2}+d(x)^{2}}\right)},
$$


where

$$
f(x)=\frac{\omega}{2 \pi}\left(\frac{d x}{d E}\right)_{E_{n}} \text { and } d(x)=(2-x)_{E_{n}}>0 .
$$

Each complex pole in Eq. (23) is given by

$$
W_{n}=E_{n}+\Delta_{n}-(i / 2) \Gamma_{n},
$$

where

$$
\begin{aligned}
& \Delta_{n}=-\frac{\omega}{2 \pi} \frac{x f(x)}{f(x)^{2}+d(x)^{2}} \quad \text { (energy shift), } \\
& \Gamma_{n}=\frac{\omega}{\pi} \frac{x d(x)}{f(x)^{2}+d(x)^{2}}>0 \quad \text { (energy width). }
\end{aligned}
$$

Note that the result for $\Gamma_{n}$ shows that this quantity is positive. This is in accordance with our previous result, ${ }^{5}$ where the potential is defined by the regions $\mathrm{O}$, I, II.

Here there is an additional pole, due to the term

$$
\frac{8 \sin \mu\left(r_{1}\right) \sin \mu\left(r_{2}\right)\left[2\left(1-i \delta^{x}\right)+i x \delta^{x}\right]}{\left[2\left(1-i \beta^{x}\right)+i x \beta^{x}\right]\left[2\left(1-i \delta^{x}\right)+i x \delta^{x}\right]-4 x^{2} \exp \left(2 \kappa_{1}\right) \beta^{x} \delta^{x}} .
$$

We distinguish the symmetric from the asymmetric case of the DWP.

\section{B. Symmetric case}

For such a potential, $\beta^{x}=\delta^{x}$. Therefore, the above term can be written as [neglecting $8 \sin \mu\left(r_{1}\right) \sin \mu\left(r_{2}\right)$ ],

$$
\frac{w}{w^{2}-q^{2}}=\frac{1}{2} \frac{1}{w-q}+\frac{1}{2} \frac{1}{w+q},
$$

where

\section{$\left(J_{3}\right)$}

$$
\begin{aligned}
& w=\left[2\left(1-i \beta^{x}\right)+i x \beta^{x}\right]=\left[2\left(1-i \delta^{x}\right)+i x \delta^{x}\right], \\
& q=2 x \exp \left(\kappa_{1}\right) \beta^{x}=2 x \exp \left(\kappa_{1}\right) \delta^{x} .
\end{aligned}
$$

We can analyze each of the two poles of Eq. (27) separately. We start with the term $(1 / 2)(1 / w-q)$. The corresponding part of the propagator is

$$
\frac{4 \sin \mu\left(r_{1}\right) \sin \mu\left(r_{2}\right)}{\left[2\left(1-i \beta^{x}\right)+i x \beta^{x}\right]-2 x \exp \left(\kappa_{1}\right) \beta^{x}} .
$$

\section{Application to the DWP with harmonic wells}

We expand the denominator around the point $\beta^{x}=-i$, that is around the eigenvalues of one well. Let us assume that the DWP consists of two HOWs. In this case, according to our previous results, ${ }^{5}$

$$
\frac{d \beta^{x}}{d E}=\frac{2 \pi}{\omega} .
$$

If we define the quantity:

$$
\left.\frac{\omega}{\pi} \frac{d\left(x e^{\kappa}\right)}{d E}\right|_{E_{n}}=g(x)
$$

the following relation is obtained:

$$
\begin{gathered}
\frac{1}{\left[2\left(1-i \beta^{x}\right)+i x \beta^{x}\right]-2 x \exp \left(\kappa_{1}\right) \beta^{x}} \\
\simeq \frac{\left(c^{-}\right)^{-1}}{\left\{E-\left(E_{n}-\Delta_{n}^{-}-i \Gamma_{n}^{-} / 2\right)\right\}} .
\end{gathered}
$$

Here,

$$
c^{-}=\frac{2 \pi}{\omega}\left(\left[f(x)-2 x \exp \left(\kappa_{1}\right)\right]+i[x-2+g(x)]\right)_{E_{n}},
$$

$$
\begin{aligned}
& \Delta_{n}^{-}=\left.\frac{\omega}{2 \pi} \frac{x f(x)+2 x \exp \left(\kappa_{1}\right)(g(x)-2)}{f(x)^{2}+4 x^{2} \exp \left(2 \kappa_{1}\right)-4 x \exp \left(\kappa_{1}\right) f(x)+(x-2)^{2}+g(x)^{2}+2(x-2) g(x)}\right|_{E_{n}}, \\
& \Gamma_{n}^{-}=\left.\frac{\omega}{\pi} \frac{2 x \exp \left(\kappa_{1}\right) f(x)-4 x^{2} \exp \left(2 \kappa_{1}\right)-x(x-2)-x g(x)}{f(x)^{2}+4 x^{2} \exp \left(2 \kappa_{1}\right)-4 x \exp \left(\kappa_{1}\right) f(x)+(x-2)^{2}+g(x)^{2}+2(x-2) g(x)}\right|_{E_{n}} .
\end{aligned}
$$

Repeating the procedure for the other pole we find:

$$
\begin{aligned}
& \frac{1}{\left[2\left(1-i \beta^{x}\right)+i x \beta^{x}\right]+2 x \exp \left(\kappa_{1}\right) \beta^{x}} \simeq \frac{\left(c^{+}\right)^{-1}}{\left\{E-\left(E_{n}-\Delta_{n}^{+}-(i / 2) \Gamma_{n}^{+}\right)\right\}}, \\
& c^{+}=\frac{2 \pi}{\omega}\left(\left[f(x)+2 x \exp \left(\kappa_{1}\right)\right]+i[x-2-g(x)]\right)_{E_{n}}, \\
& \Delta_{n}^{+}=\left.\frac{\omega}{2 \pi} \frac{x f(x)+2 x \exp \left(\kappa_{1}\right)(g(x)+2)}{f(x)^{2}+4 x^{2} \exp \left(2 \kappa_{1}\right)+4 x \exp \left(\kappa_{1}\right) f(x)+(x-2)^{2}+g(x)^{2}-2(x-2) g(x)}\right|_{E_{n}}, \\
& \Gamma_{n}^{+}=\left.\frac{\omega}{\pi} \frac{-2 x \exp \left(\kappa_{1}\right) f(x)-4 x^{2} \exp \left(2 \kappa_{1}\right)-x(x-2)+x g(x)}{f(x)^{2}+4 x^{2} \exp \left(2 \kappa_{1}\right)+4 x \exp \left(\kappa_{1}\right) f(x)+(x-2)^{2}+g(x)^{2}-2(x-2) g(x)}\right|_{E_{n}} .
\end{aligned}
$$


If we now make the realistic assumptions that

$$
f(x) \ll 1, \quad g(x) \ll 1, \quad(x-2) \simeq-2
$$$$
\text { (i.e., } x \text { is very small). }
$$

then,

$$
\begin{aligned}
& \Delta_{n}^{-} \simeq-\frac{\omega}{2 \pi} \exp \left(-\kappa_{1}\right), \quad \Delta_{n}^{+} \simeq+\frac{\omega}{2 \pi} \exp \left(-\kappa_{1}\right), \\
& \Gamma_{n}^{-}=\Gamma_{n}^{+} \simeq-\frac{\omega}{2 \pi} \exp \left(-2 \kappa_{1}\right) .
\end{aligned}
$$

At this point we stress that the above quantities as well as those that will appear below, are calculated at the eigenvalue $E_{n}$ of the initial state. For the sake of economy and brevity, we omit the corresponding indices.

Under the assumptions (31), the initial state has been split in two new states which are almost degenerate, with an energy difference $\Delta E=(\omega / \pi) \exp \left(-\kappa_{1}\right)$. This is the result of the JWKB approximation. Therefore, the herein derived formulas for the energy shift, Eqs. (29c) and (30c), represent a level of accuracy that goes beyond the JWKB approximation, since the present treatment accounts for the energy dependence of the tunneling process.

A noteworthy result is that, by considering the quantity $\kappa_{1}$ as energy dependent, the symmetry in the plus-minus quantities of the energy shift of the symmetric DWP states, Eqs. (29c) and (30c), is slightly destroyed. In other words, even if we take the denominators to be the same, (keeping only the dominant terms), the numerators do not have opposite signs, due to the presence of the much smaller terms $x f(x), 2 x \exp \left(\kappa_{1}\right) g(x)$, whose sign is the same in both cases. This fact is again connected to the energy dependence of the tunneling process, albeit of small magnitude. The imaginary parts of the two poles (29d), (30d) are not simultaneously the same, the higher one having a slightly larger rate. Of course, the dominant terms are the same for both poles.

\section{Asymmetric case}

In this case, we expect to see the poles (states) of only the well where the points $r_{1}$ and $r_{2}$ are located, shifted by the interaction with the rest of the system. Let us consider the first well (the same treatment holds for the second well), and focus on the term

$$
\frac{1}{\left[2\left(1-i \beta^{x}\right)+i x \beta^{x}\right]-\frac{4 x^{2} \exp \left(2 \kappa_{1}\right) \beta^{x} \delta^{x}}{\left[2\left(1-i \delta^{x}\right)+i x \delta^{x}\right]}}
$$

\section{E. Application to two unequal HOWs}

We expand the denominator $(D)$ around one eigenvalue, $E_{1}$, of the first well. By keeping only the most important terms, $D$ is reduced to:

$$
D \simeq x+\frac{4 i x^{2} \exp \left(2 \kappa_{1}\right) \delta^{x}\left(E_{1}\right)}{\left[2\left(1-i \delta^{x}\left(E_{1}\right)\right)+i x \delta^{x}\left(E_{1}\right)\right]}-\frac{4 i \pi}{\omega}\left(E-E_{1}\right)
$$

Based on the physics of the problem, we take

$$
x^{2} \exp \left(2 \kappa_{1}\right) \simeq \exp \left(-2 \kappa_{1}\right),
$$

and

$$
\left|-2 i \delta^{x}\right| \gg\left|i x \delta^{x}\right|
$$

Then,

$$
\begin{aligned}
D & \simeq x+\frac{4 i \exp \left(-2 \kappa_{1}\right) \delta^{x}\left(E_{1}\right)}{2\left(1-i \delta^{x}\left(E_{1}\right)\right)}-\frac{4 i \pi}{\omega}\left(E-E_{1}\right) \\
& =-\frac{2 i \pi}{\omega}\left\{\left(E-E_{1}\right)-\frac{\left(1+i \delta^{x}\left(E_{1}\right)\right)}{\left(1-i \delta^{x}\left(E_{1}\right)\right)}\right\} .
\end{aligned}
$$

We expand the denominator of the fraction of the last term around an eigenvalue $E_{2}$ of the second well, assumed to be very close to $E_{1}$. In the numerator, $E_{1}$ is simply replaced by $E_{2}$. Then,

$$
\begin{aligned}
& D \simeq \frac{4 i \pi}{\omega}\left\{\left(E-E_{1}\right)\right. \\
& \left.-\frac{\left(1+i \delta^{x}\left(E_{2}\right)\right) \exp \left(-2 \kappa_{1}\right)}{\left(1-i \delta^{x}\left(E_{2}\right)-i\left(\frac{d \delta^{x}}{d E}\right)_{E_{2}}\left(E_{1}-E_{2}\right)\right)} \frac{\omega}{4 i \pi}\right\}
\end{aligned}
$$

Since $E_{2}$ is an eigenvalue of the second well, the relation $\delta^{x}\left(E_{2}\right)=-i$ holds. [We note that Miller ${ }^{7}$ defines a quantity $n_{2}(E)$, which is related to $\delta^{x}$ as follows:

$$
\left.\delta^{x}=-\exp \left(2 i \pi n_{2}\right) .\right]
$$

Therefore, we write:

$$
D \simeq-\frac{4 i \pi}{\omega}\left\{\left(E-E_{1}\right)-\frac{\omega}{2 \pi} \frac{\exp \left(-2 \kappa_{1}\right)}{2 \pi\left(\frac{d n_{2}}{d E}\right)_{E_{2}}\left(E_{1}-E_{2}\right)}\right\} .
$$

However,

$$
\frac{2 \pi}{\omega}=\frac{d \beta^{+}}{d E}=2 \pi\left(\frac{d n_{2}}{d E}\right)_{E_{2}},
$$

and, therefore, we finally have:

$$
\begin{aligned}
D \simeq & -\frac{4 i \pi}{\omega}\left\{\left(E-E_{1}\right)\right. \\
& \left.-\frac{\exp \left(-2 \kappa_{1}\right)}{2 \pi\left(\frac{d n_{1}}{d E}\right)_{E_{1}} 2 \pi\left(\frac{d n_{2}}{d E}\right)_{E_{2}}\left(E_{1}-E_{2}\right)}\right\} .
\end{aligned}
$$

Equation (41) shows that, if the above approximations are made, the semiclassical energy shift in the asymmetric DWP is 


$$
\Delta E=\frac{\exp \left(-2 \kappa_{1}\right)}{2 \pi\left(\frac{d n_{1}}{d E}\right)_{E_{1}} 2 \pi\left(\frac{d n_{2}}{d E}\right)_{E_{2}}\left(E_{1}-E_{2}\right)} .
$$

Equation (42) is the same as the one first given by Miller [Eq. (21) of Ref. 7]. Note that in both derivations, ours and that of Ref. 7, the energies $E_{1}$ and $E_{2}$ are assumed to be close, meaning that the asymmetry of the DWP is assumed to be small.

We proceed to derive $\Delta E$ and $\Gamma$ for the DWP beyond the approximations leading to Eq. (42). To do so, we expand the denominator around an eigenvalue $E_{n}$ of the first well which is not close to an eigenvalue of the second well.

Keeping the main terms, that is making the realistic assumptions that

$$
\left|-\frac{4 i \pi}{\omega}\right| \gg\left|\frac{2 i x \pi}{\omega}\right|
$$

and

$$
\left|-2 i \delta^{x}\right| \gg\left|i x \delta^{x}\right|
$$

and ignoring powers smaller than $\exp \left(-2 \kappa_{1}\right)$, such as $x^{2} \exp \left(2 \kappa_{1}\right) f(x)$ and $x^{2} \exp \left(2 \kappa_{1}\right) x$, and putting $\delta^{x}\left(E_{n}\right)=\delta_{R}^{x}$ $+i \delta_{1}^{x}$, we end up with the relation

$$
\begin{gathered}
\frac{1}{\left[2\left(1-i \beta^{x}\right)+i x \beta^{x}\right]-\frac{4 x^{2} \exp \left(2 \kappa_{1}\right) \beta^{x} \delta^{x}}{\left[2\left(1-i \delta^{x}\right)+i x \delta^{x}\right]}} \\
\simeq \frac{d}{\left(E-E_{n}-\Delta E_{n}-i \frac{\Gamma_{n}}{2}\right)},
\end{gathered}
$$

where:

$$
\begin{aligned}
& d=-\frac{4 \pi\left\{\left(1+\delta_{R, I}\right)-2 i \delta_{R}^{x}\left(1+\delta_{I}^{x}\right)\right\}}{\omega\left\{2 \delta_{R}^{x}\left(1+\delta_{I}^{x}\right)+i\left(1+\delta_{R, I}\right)\right\}}, \\
& \Delta E_{n}=\frac{4 \pi x^{2} \exp \left(2 \kappa_{1}\right)\left\{\left(1+\delta_{R}^{x^{2}}-\delta_{I}^{x^{2}}\right) 2 \delta_{R}^{x}\left(1+\delta_{I}^{x}\right)\right\}}{\omega\left\{4 \delta_{R}^{x^{2}}\left(1+\delta_{I}^{x}\right)^{2}+\left(1+\delta_{R, I}\right)^{2}\right\}},
\end{aligned}
$$

with

$$
\delta_{R, I}=\delta_{I}^{x^{2}}-\delta_{R}^{x^{2}}+2 \delta_{I}^{x} .
$$

The above relations, where all quantities are evaluated at $E_{n}$, do not apply for $\delta_{I}^{x}=-1$, since now $E_{n}$ is an eigenvalue of the first well but not of the second. (Note that the condition for an eigenvalue of the second well is: $\delta^{x}=-i$.)

\section{$\left(J_{4}\right) . \quad$ The DWP with tunneling}

This category produces another pole, due to the term

$$
\frac{8 \sin \mu\left(r_{1}\right) \sin \mu\left(r_{2}\right)\left[2\left(1-i \delta^{x}\right)+i x \delta^{x}\right]}{\left[2\left(1-i \beta^{x}\right)+i x \beta^{x}\right]\left[2\left(1-i \delta^{x}\right)+i x \delta^{x}\right]-4 x^{2} \exp \left(2 \kappa_{1}\right) \beta^{x} \delta^{x}+\frac{8 x^{2} \exp \left(2 \kappa_{1}\right) y \beta^{x} \delta^{x}\left(1-i \delta^{x}\right)}{\left[2\left(1-i \beta^{x}\right)+i x \beta^{x}\right]\left[2\left(1-i \delta^{x}\right)+i x \delta^{x}\right]-i x y \delta^{x}}} .
$$

This is the relevant pole for the case beyond the DWP, where all regions are considered. In order to exhibit its physical significance, we rewrite Eq. (45) as follows:

$$
8 \sin \mu\left(r_{1}\right) \sin \mu\left(r_{2}\right) \frac{\eta\left(\delta^{x}, x\right)\left[\eta\left(\delta^{x}, x\right) \eta\left(\delta^{x}, y\right)-i x y \delta^{x}\right]}{\left\{\eta\left(\beta^{x}, x\right) \eta\left(\delta^{x}, x\right)-4 x^{2} \exp \left(2 \kappa_{1}\right) \beta^{x} \delta^{x}\right\}\left\{\eta\left(\delta^{x}, x\right) \eta\left(\delta^{x}, y\right)-i x y \delta^{x}\right\}+8 x^{2} \exp \left(2 \kappa_{1}\right) y \beta^{x} \delta^{x}\left(1-i \delta^{x}\right)},
$$

where the following relations hold:

$$
\begin{aligned}
& {\left[2\left(1-i \beta^{x}\right)+i x \beta^{x}\right]=\eta\left(\beta^{x}, x\right),} \\
& {\left[2\left(1-i \delta^{x}\right)+i x \delta^{x}\right]=\eta\left(\delta^{x}, x\right),} \\
& {\left[2\left(1-i \delta^{x}\right)+i y \delta^{x}\right]=\eta\left(\delta^{x}, y\right) .}
\end{aligned}
$$

We observe that if $y$ is very small, meaning that the second barrier is large, then $8 x^{2} \exp \left(2 \kappa_{1}\right) y \beta^{x} \delta^{x}\left(1-i \delta^{x}\right)$ can be considered as negligible and, after some simplifications, the poles of the symmetric DWP emerge:

$$
\frac{\eta\left(\delta^{x}, x\right)}{\eta\left(\beta^{x}, x\right) \eta\left(\delta^{x}, x\right)-4 x^{2} \exp \left(2 \kappa_{1}\right) \beta^{x} \delta^{x}}
$$

So, in this case, whether we have prepared the wave packet in one of the nonstationary states of the first well or in one of 
the stationary states of the double well, the expansion will be done around the poles of the DWP. This is reasonable since, even if we consider the first case, due to the small decay from the last barrier the system has time to equilibrate in a DWP stationary state.

If we consider that the condition $x \ll 1$ is also satisfied, then the poles of the first well emerge from the perturbation of the bound states of the first well by the two barriers, the second well and the continuum. This is seen by the fact that, after certain simplifications, a quantity proportional to $\left[1 / 2\left(1-i \beta^{x}\right)+i x \beta^{x}\right]$ remains. (This is the $J_{2}$ case.)

Given the above, in the expansion that follows we assume that the system has been prepared in a state of the first well and we examine the dependence of the new poles on the relative magnitude of the two barriers.

\section{CALCULATION OF THE COMPLEX ENERGIES OF THE FULL GREEN'S FUNCTION}

The complete problem is understood when the complex poles are extracted from the relevant fraction of the full Green's function (category $J_{4}$ ). With reference to the unperturbed model, energy shifts and widths for both the symmetric and the asymmetric cases can be found.

We start with the expression for the Green's function, excluding the regular part [Eq. (45)]. Again, we will distinguish two cases, depending on whether the two wells are the same or not.

\section{A. Symmetric DWP plus continuum}

In this case, $\beta^{x}=\delta^{x}$. We write $G_{s c}(E)$ as [without the factor $8 \sin \mu\left(r_{1}\right) \sin \mu\left(r_{2}\right)$ ]

$$
\frac{\left[2\left(1-i \beta^{x}\right)+i x \beta^{x}\right]}{\left[2\left(1-i \beta^{x}\right)+i x \beta^{x}\right]^{2}-4 x^{2} \exp \left(2 \kappa_{1}\right) \beta^{x} \beta^{x}+\frac{8 x^{2} \exp \left(2 \kappa_{1}\right) y \beta^{x} \beta^{x}\left(1-i \beta^{x}\right)}{\left[2\left(1-i \beta^{x}\right)+i x \beta^{x}\right]^{2}-i x y \beta^{x}}} .
$$

Let

$$
\zeta=\frac{8 x^{2} \exp \left(2 \kappa_{1}\right) y \beta^{x} \beta^{x}\left(1-i \beta^{x}\right)}{\left[2\left(1-i \beta^{x}\right)+i x \beta^{x}\right]^{2}-i x y \beta^{x}} .
$$

We put:

$$
4 x^{2} \exp \left(2 \kappa_{1}\right) \beta^{x} \beta^{x}-\zeta=\varepsilon^{2},
$$

where

$$
\varepsilon=\rho e^{i \varphi}
$$

with $\rho$ and $\varphi$ being real numbers. Thus expression (47) takes the form:

$$
\begin{aligned}
\frac{\left[2\left(1-i \beta^{x}\right)+i x \beta^{x}\right]}{\left[2\left(1-i \beta^{x}\right)+i x \beta^{x}\right]^{2}-\varepsilon^{2}}= & \frac{1}{2} \frac{1}{\left[2\left(1-i \beta^{x}\right)+i x \beta^{x}\right]-\varepsilon} \\
& +\frac{1}{2} \frac{1}{\left[2\left(1-i \beta^{x}\right)+i x \beta^{x}\right]+\varepsilon} .
\end{aligned}
$$

We observe that if the quantity $\zeta$ is zero, then the problem is reduced to that of the symmetric DWP. The condition for this to occur is for the quantity $y$ to become zero, i.e., to have the situation of an infinite second barrier.

We turn to the first term of Eq. (49) and again focus on the denominator. Expansion around the eigenvalues of the harmonic bound state problem [Eq. (28b)] of the first well yields:

$$
\begin{aligned}
& {\left[2\left(1-i \beta^{x}\right)+i x \beta^{x}\right]-\varepsilon} \\
& \simeq x+2 i x \exp \left(\kappa_{1}\right)+\left(-2 i \frac{2 \pi}{\omega}+\frac{d x}{d E}+i x \frac{2 \pi}{\omega}-\frac{d \varepsilon}{d E}\right) \\
& \quad \times\left(E-E_{n}\right) .
\end{aligned}
$$

We define the quantity

$$
t(x)=\left.\frac{\omega}{2 \pi} \frac{d\left(x^{2} \exp \left(2 \kappa_{1}\right)\right)}{d E}\right|_{E_{n}}
$$

and carry out algebra so as to obtain:

$$
\frac{1}{2} \frac{1}{\left[2\left(1-i \beta^{x}\right)+i x \beta^{x}\right]-\varepsilon} \simeq \frac{\left(c^{-}\right)^{-1}}{E-\left[E_{n}-\Delta E_{n}^{-}-i \frac{\Gamma_{n}^{-}}{2}\right]}
$$

where:

$$
\begin{aligned}
c^{-}= & \frac{4 \pi}{\omega}\left\{\left(f(x)-2 x \exp \left(\kappa_{1}\right)+\frac{2 y \exp \left(\kappa_{1}\right)}{(y-x)}\right)\right. \\
& \left.+i\left(x-2+\frac{t(x) \exp \left(-\kappa_{1}\right)}{x}\right)\right\},
\end{aligned}
$$




$$
\begin{aligned}
& \Delta E_{n}^{-}=\frac{\omega}{2 \pi} \frac{\left(f(x) x+\frac{2 x y \exp \left(\kappa_{1}\right)}{(y-x)}+2 x \exp \left(\kappa_{1}\right)\left[\frac{t(x) \exp \left(-\kappa_{1}\right)}{x}-2\right]\right)}{\left(f(x)-2 x \exp \left(\kappa_{1}\right)+\frac{2 y \exp \left(\kappa_{1}\right)}{(y-x)}\right)^{2}+\left(x-2+\frac{t(x) \exp \left(-\kappa_{1}\right)}{x}\right)^{2},} \\
& \frac{\Gamma_{n}^{-}}{2}=\frac{\omega}{2 \pi} \frac{\left(\frac{4 x y \exp \left(2 \kappa_{1}\right)}{(y-x)}+2 f(x) \exp \left(\kappa_{1}\right) x-4 x^{2} \exp \left(2 \kappa_{1}\right)-x\left[x-2+\frac{t(x) \exp \left(-\kappa_{1}\right)}{x}\right]\right)}{\left(f(x)-2 x \exp \left(\kappa_{1}\right)+\frac{2 y \exp \left(\kappa_{1}\right)}{(y-x)}\right)^{2}+\left(x-2+\frac{t(x) \exp \left(-\kappa_{1}\right)}{x}\right)^{2}} .
\end{aligned}
$$

We repeat the procedure for the second pole:

$$
\left[2\left(1-i \beta^{x}\right)+i x \beta^{x}\right]+\varepsilon \simeq x-2 i x \exp \left(\kappa_{1}\right)+\left(-2 i \frac{2 \pi}{\omega}+f(x)+i x \frac{2 \pi}{\omega}+\frac{d \varepsilon}{d E}\right)\left(E-E_{n}\right),
$$

from which

$$
\frac{1}{2} \frac{1}{\left[2\left(1-i \beta^{x}\right)+i x \beta^{x}\right]+\varepsilon} \simeq \frac{\left(c^{+}\right)^{-1}}{E-\left[E_{n}-\Delta E_{n}^{+}-i \frac{\Gamma_{n}^{+}}{2}\right]},
$$

where

$$
\begin{aligned}
& c^{+}=\frac{4 \pi}{\omega}\left\{\left(f(x)+2 x \exp \left(\kappa_{1}\right)-\frac{2 y \exp \left(\kappa_{1}\right)}{(y-x)}\right)+i\left(x-2-\frac{t(x) \exp \left(-\kappa_{1}\right)}{x}\right)\right\}, \\
& \Delta E_{n}^{+}=\frac{\omega}{2 \pi} \frac{\left(f(x) x-\frac{2 x y \exp \left(\kappa_{1}\right)}{(y-x)}+2 x \exp \left(\kappa_{1}\right)\left[2+\frac{t(x) \exp \left(-\kappa_{1}\right)}{x}\right]\right)}{\left(f(x)+2 x \exp \left(\kappa_{1}\right)+\frac{2 y \exp \left(\kappa_{1}\right)}{(y-x)}\right)^{2}+\left(x-2-\frac{t(x) \exp \left(-\kappa_{1}\right)}{x}\right)^{2},} \\
& \frac{\Gamma_{n}^{+}}{2}=\frac{\omega}{2 \pi} \frac{\left(\frac{4 x y \exp \left(2 \kappa_{1}\right)}{(y-x)}-2 f(x) \exp \left(\kappa_{1}\right) x-4 x^{2} \exp \left(2 \kappa_{1}\right)-x\left[x-2-\frac{t(x) \exp \left(-\kappa_{1}\right)}{x}\right]\right)}{\left(f(x)+2 x \exp \left(\kappa_{1}\right)-\frac{2 y \exp \left(\kappa_{1}\right)}{(y-x)}\right)^{2}+\left(x-2-\frac{t(x) \exp \left(-\kappa_{1}\right)}{x}\right)^{2}} .
\end{aligned}
$$

\section{B. Return to the Green's function $G_{s c}(E)$}

At this point, we can write the form for the full $G_{s c}(E)$. It consists of the regular part, which originates from region I, and from the sum of the two terms, Eqs. (51a), (53), that contain poles [including the factor $8 \sin \mu\left(r_{1}\right) \sin \mu\left(r_{2}\right)$ ]:

$$
G_{s c}(E)=G_{\text {regular }}(E)+G_{\text {pole }}^{+}(E)+G_{\text {pole }}^{-}(E),
$$

where

$$
\begin{aligned}
& G_{\text {regular }}(E)=\frac{-i}{\pi} \sum_{n=0}^{n_{\max }} A_{n}, \\
& G_{\text {pole }}^{+}(E)=\frac{2}{\pi} \sum_{n=0}^{n_{\max }} A_{n}^{+} \frac{1}{E-W_{n}^{+}}, \\
& G_{\text {pole }}^{-}(E)=\frac{2}{\pi} \sum_{n=0}^{n_{\max }} A_{n}^{-} \frac{1}{E-W_{n}^{-}},
\end{aligned}
$$

with the definitions

$$
\begin{aligned}
& A_{n}=\frac{\sin \mu_{n}\left(r_{1}\right) \exp \left[-i \mu_{n}\left(r_{2}\right)\right]}{\sqrt{E_{n}-V\left(r_{1}\right)} \sqrt{E_{n}-V\left(r_{2}\right)}}, \\
& A_{n}^{+}=\frac{\left(c_{n}^{+}\right)^{-1}}{\sqrt{E_{n}-V\left(r_{1}\right)} \sqrt{E_{n}-V\left(r_{2}\right)}} \sin \mu_{n}\left(r_{1}\right) \sin \mu_{n}\left(r_{2}\right),
\end{aligned}
$$

$$
A_{n}^{-}=\frac{\left(c_{n}^{-}\right)^{-1}}{\sqrt{E_{n}-V\left(r_{1}\right)} \sqrt{E_{n}-V\left(r_{2}\right)}} \sin \mu_{n}\left(r_{1}\right) \sin \mu_{n}\left(r_{2}\right),
$$

$$
W_{n}^{+}=E_{n}-\Delta E_{n}^{+}-i \frac{\Gamma_{n}^{+}}{2}
$$

$W_{n}^{-}=E_{n}-\Delta E_{n}^{-}-i \frac{\Gamma_{n}^{-}}{2}$. 


\section{Asymmetric DWP plus continuum}

For this case, the Green's function of Eq. (45) is written as

$$
\overline{\left[2\left(1-i \beta^{x}\right)+i x \beta^{x}\right]-\frac{4 x^{2} \exp \left(2 \kappa_{1}\right) \beta^{x} \delta^{x}}{\left[2\left(1-i \delta^{x}\right)+i x \delta^{x}\right]}+\frac{1}{\left[2\left(1-i \beta^{x}\right)+i x \beta^{x}\right]\left[2\left(1-i \delta^{x}\right)+i x \delta^{x}\right]^{2}-i x y \delta^{x}\left[2\left(1-i \delta^{x}\right)+i x \delta^{x}\right]}},
$$

which can be rewritten as

$$
\frac{1}{2\left(1-i \beta^{x}\right)+\Omega_{1}\left(e^{-2 \kappa_{1}} ; \beta^{x}\right)+\Omega_{2}\left(e^{-2 \kappa_{1}} ; \beta^{x}, \delta^{x}\right)+\Omega_{3}\left(e^{-2 \kappa_{1}}, e^{-2 \kappa_{2}} ; \beta^{x}, \delta^{x}\right)} .
$$

The quantities $\Omega_{\kappa}$ are defined as follows:

$$
\begin{aligned}
& \Omega_{1}\left(e^{-2 \kappa_{1}} ; \beta^{x}\right)=i x \beta^{x}, \\
& \Omega_{2}\left(e^{-2 \kappa_{1}} ; \beta^{x}, \delta^{x}\right)=-\frac{4 x^{2} \exp \left(2 \kappa_{1}\right) \beta^{x} \delta^{x}}{\left[2\left(1-i \delta^{x}\right)+i x \delta^{x}\right]}, \\
& \Omega_{3}\left(e^{-2 \kappa_{1}}, e^{-2 \kappa_{2}} ; \beta^{x}, \delta^{x}\right)=\frac{8 x^{2} \exp \left(2 \kappa_{1}\right) y \beta^{x} \delta^{x}\left(1-i \delta^{x}\right)}{\left[2\left(1-i \beta^{x}\right)+i x \beta^{x}\right]\left[2\left(1-i \delta^{x}\right)+i x \delta^{x}\right]^{2}-i x y \delta^{x}\left[2\left(1-i \delta^{x}\right)+i x \delta^{x}\right]} .
\end{aligned}
$$

In this form, one can distinguish the perturbation that is applied to the bound states of the first well by the first barrier (59a), by the first barrier and the second well (59b), and finally by the two barriers and the second well (59c). When these terms and their derivatives are evaluated at the eigenvalues of the first well, they give rise to complex numbers. So, using the index $R$ for the real part and the index $I$ for the imaginary one, we obtain for the denominator (den):

$$
\begin{aligned}
& \operatorname{den} \simeq c\left\{E-E_{n}+\frac{\omega}{2 \pi}\right. \\
& \times \frac{\left\{\left(x+\Omega_{2 R}+\Omega_{3 R}\right)+i\left(\Omega_{21}+\Omega_{31}\right)\right\}\left\{\left(f(x)+\frac{\omega}{2 \pi} \frac{d \Omega_{2 R}}{d E}+\frac{\omega}{2 \pi} \frac{d \Omega_{3 R}}{d E}\right)-i\left(x-2+\frac{\omega}{2 \pi} \frac{d \Omega_{21}}{d E}+\frac{\omega}{2 \pi} \frac{d \Omega_{31}}{d E}\right)\right\}}{\left(f(x)+\frac{\omega}{2 \pi} \frac{d \Omega_{2 R}}{d E}+\frac{\omega}{2 \pi} \frac{d \Omega_{3 R}}{d E}\right)^{2}+\left(x-2+\frac{\omega}{2 \pi} \frac{d \Omega_{21}}{d E}+\frac{\omega}{2 \pi} \frac{d \Omega_{31}}{d E}\right)^{2}},
\end{aligned}
$$

where the quantity $c$ is defined as:

$$
c=\frac{2 \pi}{\omega}\left\{\left(f(x)+\frac{\omega}{2 \pi} \frac{d \Omega_{2 R}}{d E}+\frac{\omega}{2 \pi} \frac{d \Omega_{3 R}}{d E}\right)+i\left(x-2+\frac{\omega}{2 \pi} \frac{d \Omega_{21}}{d E}+\frac{\omega}{2 \pi} \frac{d \Omega_{31}}{d E}\right)\right\} .
$$

It follows that, in this case,

$$
\begin{aligned}
& \Delta E_{n}=\frac{\omega}{2 \pi} \frac{\left(x+\Omega_{2 R}+\Omega_{3 R}\right)\left(f(x)+\frac{\omega}{2 \pi} \frac{d \Omega_{2 R}}{d E}+\frac{\omega}{2 \pi} \frac{d \Omega_{3 R}}{d E}\right)+\left(\Omega_{2 I}+\Omega_{3 I}\right)\left(x-2+\frac{\omega}{2 \pi} \frac{d \Omega_{2 I}}{d E}+\frac{\omega}{2 \pi} \frac{d \Omega_{3 I}}{d E}\right)}{\left(f(x)+\frac{\omega}{2 \pi} \frac{d \Omega_{2 R}}{d E}+\frac{\omega}{2 \pi} \frac{d \Omega_{3 R}}{d E}\right)^{2}+\left(x-2+\frac{\omega}{2 \pi} \frac{d \Omega_{2 I}}{d E}+\frac{\omega}{2 \pi} \frac{d \Omega_{3 I}}{d E}\right)^{2}}, \\
& \frac{\Gamma_{n}}{2}=\frac{\omega}{2 \pi} \frac{\left(\Omega_{2 I}+\Omega_{3 I}\right)\left(f(x)+\frac{\omega}{2 \pi} \frac{d \Omega_{2 R}}{d E}+\frac{\omega}{2 \pi} \frac{d \Omega_{3 R}}{d E}\right)-\left(x+\Omega_{2 R}+\Omega_{3 R}\right)\left(x-2+\frac{\omega}{2 \pi} \frac{d \Omega_{2 I}}{d E}+\frac{\omega}{2 \pi} \frac{d \Omega_{3 I}}{d E}\right)}{\left(f(x)+\frac{\omega}{2 \pi} \frac{d \Omega_{2 R}}{d E}+\frac{\omega}{2 \pi} \frac{d \Omega_{3 R}}{d E}\right)^{2}+\left(x-2+\frac{\omega}{2 \pi} \frac{d \Omega_{2 I}}{d E}+\frac{\omega}{2 \pi} \frac{d \Omega_{3 I}}{d E}\right)^{2}} .
\end{aligned}
$$

\section{THE DEPENDENCE OF $\Gamma_{n}$ ON THE RELATIVE MAGNITUDES OF THE TWO BARRIERS FOR THE CASE OF EQUAL WELLS}

For both poles in Eqs. (51d) and (53d), the dependence of $\Gamma_{n} / 2$ on the second barrier, that is on the quantity $y$, is expressed in terms of the same quantities in both the numerator and the denominator. This is reasonable, since the second barrier did not cause any splitting. We recall that the creation of the two poles is due to the first barrier. In the case of the DWP, although the relations for $\Gamma_{n}^{+}$and $\Gamma_{n}^{-}$are different, the 
dominant terms are the same. This is why, in the limit of a large barrier, we obtain the correct JWKB result-same in both cases.

We now raise the question of the variation of $\Gamma_{n}$ as the relative size of the two barriers changes. We assume that the two wells are the same, we keep the first barrier constant and we change the second according to $y=\lambda x$. [The symbol $\lambda$ here is not the function $\lambda(r)$ of eq. (7).] In this way, $\lambda$ is a variable for the second barrier, before decay to the continuum. For example, it can be related to an electric field, E. i.e., $\lambda \equiv \lambda(\mathbf{E})$. We will show that the width changes sign as $\lambda$ becomes larger. This is caused by the operation of two "mechanisms" concerning the dynamics of the system, the one reflecting the preparation of a virtual or real two-level equilibrium state, and the other reflecting the decay into the continuum. The first mechanism produces a positive imaginary part of the complex self-energy, while the second one produces a negative one. The latter mechanism dominates over the first one as $\lambda$ increases.

We discuss the following five cases for the value of the parameter $\lambda$, each of which describes a different physical situation.

(i) If $\lambda \cong 0$, (for a finite value of $x$ ), the second barrier becomes infinite. Let us assume, without loss of generality, the harmonic oscillator potential. Then, both $\Gamma_{n}^{+}$and $\Gamma_{n}^{-}$ tend to the quantity

$$
\Gamma_{n}^{-}=\Gamma_{n}^{+} \simeq-\frac{\omega}{2 \pi} \exp \left(-2 \kappa_{1}\right) .
$$

The absolute value of the quantity $\Gamma_{n} / 2$ is the imaginary part of the complex self-energy, and therefore the solution does not lead to exponential decay, since it represents a bound system. In this case, the physical meaning of $\Gamma$ is that it represents the result of the interaction via which the system is taken from a state of the first well to a bound state of the double well. Hence, $\Gamma$ is equal to the rate with which the system approaches the two-level equilibrium state. At the end of the interaction, the system is led to an oscillation between the states of the two wells.

(ii) If $0<\lambda<1$, we have the case where the second barrier is larger in magnitude than the first one, but not infinite. In this case, there will be two different processes. The finiteness of the second barrier allows exponential decay, as soon as $\lambda$ is larger than zero. At the same time, the system tends to equilibrate to a virtual two-level stationary state, as if propagation were forbidden after the last turning point of the second well. Hence, the terms constituting the negative part of the imaginary self-energy correspond to the exponential decay, whilst those that constitute the positive part correspond to the approach to a virtual two-level equilibrium state. As $\lambda$ increases, but without affecting the region of the second well under $E_{n}$, meaning that the two wells remain symmetric, two things happen:

(a) The positive part of the imaginary self-energy remains the same, since in our approximation the preparation of the virtual two-level stationary state concerns only the two wells and the in-between barrier.

(b) The absolute value of the negative part becomes larger, since the system is now allowed to decay into the continuum. Therefore, we expect that the imaginary part of the self-energy must be a positive number whose magnitude decreases as $\lambda$ increases. The fact that it is positive implies that the rate with which the system approaches the virtual two-level stationary state is larger than the rate with which the system decays into the continuum, while the fact that its magnitude decreases implies that the second rate (exponential decay), tends to dominate the first.

Indeed, for $\lambda=1 / 3$ we find that the imaginary part approximately equals $(\omega / \pi) \exp \left(-2 \kappa_{1}\right)$, while for $\lambda=1 / 2$ it approximately equals $(\omega / 2 \pi) \exp \left(-2 \kappa_{1}\right)$, which means a decrease by half its magnitude.

We also find terms with opposite sign for the two poles, and so these terms are related to the splitting effect that characterizes the symmetric DWP. They are negligible if the barriers are treated within an energy-independent scheme.

(iii) If $1<\lambda<\infty$, we have the case where the second barrier has become smaller than the first one, and so we expect the imaginary part of the self-energy to be a negative number, with increasing absolute value tending to the limit $-(\omega / 2 \pi) \exp \left(-2 \kappa_{1}\right)$. Now, the rate with which the system decays exponentially is larger than the one with which the system tries to equilibrate, and the latter approaches zero as $\lambda$ increases. Indeed for $\lambda=2$ we find that the imaginary part equals $-(\omega / 4 \pi) \exp \left(-2 \kappa_{1}\right)$, while for $\lambda=3$ the imaginary part equals $-(\omega / 3 \pi) \exp \left(-2 \kappa_{1}\right)$. This implies an increase of $4 / 3$ in absolute magnitude.

(iv) On the other hand, if $\lambda \rightarrow \infty$, meaning that the second barrier is much smaller than the first, then both of these quantities tend to

$$
\Gamma_{n}^{-}=\Gamma_{n}^{+} \simeq \frac{\omega}{\pi} \exp \left(-2 \kappa_{1}\right)
$$

According to (iii) the opposite of this quantity (twice the imaginary part) is the result of the sum of the positive quantity $(\omega / 2 \pi) \exp \left(-2 \kappa_{1}\right)$ and a negative one corresponding to exponential decay. So the rate with which the system decays exponentially is equal to $(3 \omega / 2 \pi) \exp \left(-2 \kappa_{1}\right)$. This result holds if one assumes that even though the second barrier becomes much smaller than the first one, the second well is not affected and still remains equal to the first. If this is not the case, then we would have only $J_{2}$ in the Green's function and, according to Eq. (25c), this would give approximately a quantity equal to $-(\omega / 2 \pi) \exp \left(-2 \kappa_{1}\right)$ for twice the imaginary part, which is the one predicted by the JWKB approximation.

(v) If $\lambda=1$, which corresponds to the case where the two barriers are equal, it is easy to see that the imaginary part of the self-energy tends to zero as $-(\omega / 2 \pi)(\lambda-1) /$ $\lambda \exp \left(-2 \kappa_{1}\right)$, changing sign at $\lambda=1$, which corresponds to the situation where the two rates are equal.

\section{THE DEPENDENCE OF $\Delta E_{n}$ ON THE RELATIVE MAGNITUDES OF THE TWO BARRIERS FOR THE CASE OF EQUAL WELLS}

We now examine the problem of what happens to $\Delta E_{n}$ as the relative size of the two barriers changes. This is an 
interesting question, related to the fact that, for the symmetric case, where the doublet splitting leads to the notion of an inversion frequency, as in the standard case of ammonia, we expect that the results ought to have physical significance. The same relation for the barriers, $y=\lambda x$, is used.

It is again evident that in this case as well, the dominant terms that are crucial for the qualitative description of the model are those containing the quantity $y$, that is the contribution of the second barrier. Yet, there is a basic difference that we meet in this case as compared with the behavior of $\Gamma_{n}^{-}$and $\Gamma_{n}^{+}$, and it comes from the structure of the numerator. Now, although the dominant term in the numerator is the same in absolute value for both poles, its sign changes. This reflects the known situation of the splitting in the energies of the DWP. A physical consequence of this fact ought to be the change of the period of oscillation of ammonialike states.

We discuss the following cases corresponding to different values of $\lambda$ :

(i) If $\lambda \simeq 0$, we have a nearly infinite second barrier. Then,

$$
\Delta E_{n}^{+} \simeq+\frac{\omega}{2 \pi} \exp \left(-\kappa_{1}\right) \text { and } \Delta E_{n}^{-} \simeq-\frac{\omega}{2 \pi} \exp \left(-\kappa_{1}\right)
$$

in agreement with the JWKB result. ${ }^{1,2}$

(ii) If $0<\lambda \ll 1$, meaning that the second barrier is much larger than the first one but not infinite, say $y \sim \exp \left(-3 \kappa_{1}\right)$, then we find that

$\Delta E_{n}^{+} \simeq+\frac{\omega}{2 \pi} \frac{\exp \left(-\kappa_{1}\right)}{2}$ and $\Delta E_{n}^{-} \simeq-\frac{\omega}{2 \pi} \frac{\exp \left(-\kappa_{1}\right)}{2}$

(iii) If $\lambda$ is of order 1 , say $\lambda=1 / 2$, where the first barrier is slightly smaller than the second one, then

$$
\Delta E_{n}^{+} \simeq+\frac{\omega}{2 \pi} \frac{3 \exp \left(-3 \kappa_{1}\right)}{2}
$$

and

$$
\Delta E_{n}^{-} \simeq-\frac{\omega}{2 \pi} \frac{3 \exp \left(-3 \kappa_{1}\right)}{2} .
$$

So we observe that with the decrease of the second barrier there is a drastic reduction of the size of energy level shifts. The reason for this fact is the following: Since the first barrier becomes infinite around $\lambda \approx 0$, the increase of the quantity $y$, that is the reduction of the second barrier, has as a consequence the reduction of the degree of symmetry characterizing the two wells. In other words, the degree of perturbation of the eigenvalues is an increasing function of the degree of symmetry. This is in harmony with the result of the bound state problem of the DWP discussed above.

(iv) If $\lambda=1$ which is the limit where the two barriers $x$ and $y$ are the same, we observe that $\Delta E_{n}^{+}$and $\Delta E_{n}^{-}$tend to zero as

$$
\mp \frac{\omega}{2 \pi} \frac{(y-x)}{2} \exp \left(-\kappa_{1}\right) \text {. }
$$

This behavior is known for the periodic potential from the work of Holstein. ${ }^{9}$ Holstein's results show that a periodic potential has an infinity of poles, whose positions are given by those of the initial well shifted by a quantity proportional to $\cos \theta \exp (-\kappa)$, where the angle $\theta$ is in the interval $(-\pi, \pi)$. In our case, we do not have a periodic potential. Nevertheless, the finite number of poles are characterized by the same form, with $\theta$ having a specific value. We choose $y \cong x$ and not $y=x$ (as in a periodic potential), since in the latter case, the result would be zero. From the fraction that describes the energy shift we kept only the dominant terms. Therefore, by letting $y \cong x$ we are led to considering the additional terms which cause a small inequality. The poles are very close to the initial eigenvalues, which corresponds to values for the angle $\theta$ of $\pi / 2$.

(v) Finally, for the case where $\lambda \gg 1$, that is where the second barrier is much smaller than the first one, we find that

$$
\Delta E_{n}^{+} \simeq+\frac{\omega}{2 \pi} \frac{\exp \left(-3 \kappa_{1}\right)}{2}
$$

and

$$
\Delta E_{n}^{-} \simeq-\frac{\omega}{2 \pi} \frac{\exp \left(-3 \kappa_{1}\right)}{2}
$$

(vi) An interesting conclusion coming from the above results is that the signs of the energy shifts corresponding to the two poles remain the same, independently of the value of $\lambda$ (i.e., of the magnitude of the second barrier), except for a small region between values 1 and 2, where the two poles interchange their sign.

\section{GREEN'S FUNCTION FOR TRANSITION FROM ONE WELL TO THE OTHER}

Given Fig. 1, we may have propagation from region I to III without, or with the participation of region IV.

\section{A. Regions I, II, and III}

The contribution to the propagator of these paths is

$$
L_{1}=\left(\mathrm{I}-\mathrm{II}^{*}\right)^{r_{1} / \gamma} S\{\mathrm{I}, \mathrm{II}, \mathrm{III} ; \beta, \gamma\} A_{\gamma r_{2}}^{\mathrm{III}},
$$

where

$$
S\{\mathrm{I}, \mathrm{II}, \mathrm{III} ; \beta, \gamma\}=\frac{1+\left(\mathrm{III}-\mathrm{II}^{*}\right)^{\gamma / \gamma}}{1-\left(\mathrm{III}-\mathrm{II}^{*}\right)^{\gamma / \beta}\left(\mathrm{I}-\mathrm{II}^{*}\right)^{\beta / \gamma}} .
$$

Finally:

$$
L_{1}=\frac{A_{r_{1} \beta}^{\mathrm{I}} A_{\beta \gamma}^{\mathrm{II}} A_{\gamma r_{2}}^{\mathrm{III}}}{\left(1-A_{\beta \beta}^{\mathrm{I}} A_{\beta \beta}^{\mathrm{II}}\right)\left(1-A_{\gamma \gamma}^{\mathrm{III}} A_{\gamma \gamma}^{\mathrm{II}}\right)-A_{\beta \beta}^{\mathrm{I}} A_{\beta \gamma}^{\mathrm{II}} A_{\gamma \beta}^{\mathrm{II}} A_{\gamma \gamma}^{\mathrm{III}}} .
$$

By defining the function $\zeta(r)=\lambda(r)-\lambda(\delta)+\pi / 4, L_{1}$ becomes 


$$
L_{1}=2 \sin \mu\left(r_{1}\right) \sin \zeta\left(r_{2}\right) \frac{2 \sqrt{\beta^{x} \delta^{x}} x \exp \left(\kappa_{1}\right)}{\left[2\left(1-i \beta^{x}\right)+i x \beta^{x}\right]\left[2\left(1-i \delta^{x}\right)+i x \delta^{x}\right]-4 x^{2} \exp \left(2 \kappa_{1}\right) \beta^{x} \delta^{x}}
$$

We observe that Eq. (68) is of the form

$$
-\frac{a}{b^{2}-a^{2}}=\frac{1}{2} \frac{1}{b-a}-\frac{1}{2} \frac{1}{b+a},
$$

where

$$
a=2\left(1-i \beta^{x}\right)+i x \beta^{x}=2\left(1-i \delta^{x}\right)+i x \delta^{x}
$$

and

$$
b=2 \beta^{x} x \exp \left(\kappa_{1}\right)=2 \delta^{x} x \exp \left(\kappa_{1}\right),
$$

provided that the wells are symmetric. Therefore, taking into account the previous analysis, this part of the Green's function will exhibit the poles of the symmetric DWP. The crucial difference comes from the change in sign of the second fraction, a fact that produces the opposite phase for the oscillation.

\section{B. Regions I, II, III, and IV}

We now have three additional subcategories, which have as their multiplicative factor the term (I-II-III* $)^{r_{1} / \delta}$. This factor expresses the fact that since region IV is included in the propagation, the point $\delta$ must necessarily be reached. The subcategories are the following:

(a) After point $\delta$, we include only regions IV and III. The contribution of this subcategory is

$$
L_{2 \alpha}=(\mathrm{I}-\mathrm{II}-\mathrm{III} *)^{r_{1} / \delta}\left(\mathrm{IV}-\mathrm{III}^{*}\right)^{\delta / r_{2}} .
$$

(b) After point $\delta$, we include only regions IV, III, and II. The contribution is

$$
\begin{aligned}
L_{2 \beta}= & \left.(\mathrm{I}-\mathrm{II}-\mathrm{III})^{*}\right)^{r_{1} / \delta} \\
& \times \frac{\left(\mathrm{IV}-\mathrm{III}^{*}\right)^{\delta / \gamma}\left\{(\mathrm{II}-\mathrm{III} *)^{\gamma / r_{2}}+\left(\mathrm{IV}^{-\mathrm{III}^{*}}\right)^{\delta / r_{2}}\left(\mathrm{II}^{\mathrm{III}}{ }^{*}\right)^{\gamma / \delta}\right\}}{1-\left(\mathrm{IV}-\mathrm{III}^{*}\right)^{\delta / \gamma}\left(\mathrm{II}-\mathrm{III}^{*}\right)^{\gamma / \delta}} .
\end{aligned}
$$

(c) After point $\delta$, all regions, IV, III, II, and I, are included. The contribution is

$$
\begin{aligned}
& L_{2 \gamma}=\frac{\left(\text { I-II-III }{ }^{*}\right)^{r_{1} / \delta}(\text { IV-III-II* })^{\delta / \beta}}{1-\left(\text { I-II-III }^{*}\right)^{\beta / \delta}(\text { IV-III-II })^{\delta / \beta}}\left\{(\text { I-II-III* })^{\beta / r_{2}}\right. \\
& \left.+(\text { I-II-III* })^{\beta / \delta}\left[L_{2 \beta} /(\text { I-II-III* })^{r_{1} / \delta}\right]\right\} .
\end{aligned}
$$

Doing the algebra, we end up with the result:

$$
L_{2}=L_{2 \alpha}+L_{2 \beta}+L_{2 \gamma}=R\left(r_{1}\right) S\left(r_{2}\right) \frac{\left[2\left(1-i \delta^{x}\right)+i x \delta^{x}\right]}{D},
$$

where the symbols are defined as:

$$
\begin{aligned}
S\left(r_{2}\right)= & {\left[2\left(1-i \beta^{x}\right)+i x \beta^{x}\right]\left\{\operatorname { s i n } \xi ( r _ { 2 } ) \left[2\left(1-i \delta^{x}\right)\right.\right.} \\
& \left.\left.+i x \delta^{x}\right]-i \sin \zeta\left(r_{2}\right) \sqrt{\delta} x\right\} \\
& +2 x^{2} \exp \left(\kappa_{1}\right) \beta^{x} \sqrt{\delta^{x}}\left[i \sin \zeta\left(r_{2}\right)\right. \\
& \left.-2 \sqrt{\delta^{x}} \exp \left(\kappa_{1}\right) \sin \xi\left(r_{2}\right)\right]
\end{aligned}
$$

$$
\begin{aligned}
& R\left(r_{1}\right)=-16 \sqrt{\beta^{x}} \delta^{x} \sin \mu\left(r_{1}\right) x y \exp \left(\kappa_{1}\right), \\
& D=q\left(\beta^{x}, \delta^{x}, x\right)\left\{q\left(\beta^{x}, \delta^{x}, x\right) w\left(\delta^{x}, x, y\right)\right. \\
& \left.+8 \beta^{x} \delta^{x} x^{2} y \exp \left(2 \kappa_{1}\right)\left(1-i \delta^{x}\right)\right\}, \\
& q\left(\beta^{x}, \delta^{x}, x\right)=\left[2\left(1-i \beta^{x}\right)+i x \beta^{x}\right]\left[2\left(1-i \delta^{x}\right)+i x \delta^{x}\right] \\
& -4 x^{2} \exp \left(2 \kappa_{1}\right) \beta^{x} \delta^{x}, \\
& w\left(\delta^{x}, x, y\right)=\left[2\left(1-i \delta^{x}\right)+i x \delta^{x}\right]\left[2\left(1-i \delta^{x}\right)+i y \delta^{x}\right] \\
& -i \delta^{x} x y \text {. }
\end{aligned}
$$

Therefore, provided all quantities that are related to the barriers tend to zero, this contribution exhibits three different poles, of which two constitute a double one. The double pole is created by the two wells coupled via the first barrier. The simple pole is that of the second well coupled to the second barrier.

\section{A NOTE ON PREVIOUS RELEVANT PAPERS ON THE DWP}

Even though the problem treated here is onedimensional, it does not lack physical relevance, as explained in the Introduction, especially since it includes decay into the continuum. Of course, it is a desideratum for this path-integral formalism, which goes beyond the level of the JWKB approximation, to be proven practical in the case of multidimensional potentials as well. (For treatments of aspects of multidimensional tunneling, see, e.g., Refs. 22-24.)

As regards the approach followed here, it is noteworthy that there have been earlier treatments that have analyzed aspects of the problem of the DWP (without the dissipative part), in terms of the trace of the resolvent operator or of the Green's function. We have already referred to, and compared with, the works of Miller ${ }^{7}$ and of Holstein. ${ }^{8,9}$ We should also cite the works of Strunz ${ }^{25}$ and of Andrade et al. ${ }^{26}$ both of which were brought to our attention after submission of the manuscript. Strunz ${ }^{25}$ derived a Green's function for the onedimensional multiple well potential [his Eq. (11)], using graph theory and the transfer matrix method. The barriers were taken as inverted parabolas. Contrary to the present treatment of the DWP, no practical formulas for the real and the imaginary parts of the complex energies were produced. Andrade et ll $^{26}$ produced a solution for a semiclassical Green's function with a pole structure analogous to ours. Their approach has in common with our treatment the characteristic of a building up methodology. In our case, all amplitudes and coefficients are produced analytically. In addition, the present development is transparent as regards the required computational procedures, including the imaginary parts of the poles. 


\section{CONCLUSION}

We have shown how to obtain the semiclassical energydependent Green's function, $G_{s c}(E)$, Eq. (18), for the potential of Fig. 1, and subsequently its complex poles using a practical formalism of path integrals. Analysis of the structure of $G_{s c}(E)$ reveals basic aspects of the dynamics of this system.

The building-up methodology that was adopted also allowed the investigation of aspects of the physics of the double well potential (DWP). The preparation of the system occurs in the left well and complex energies emerge naturally due to tunneling.

An interesting result for the DWP, not previously revealed in treatments at the JWKB level, ${ }^{1,2,7}$ is the appearance of an imaginary part connected to the system's duration of equilibration [Eqs. (29c), (30c), (44c)]. The fact that in a bound state problem, namely that of the DWP, there appear complex poles, has to do with the implied time evolution of the system from a nonstationary state of the first harmonic well, where it is prepared, to a final eigenstate of the bound DWP.

Formulas beyond the JWKB approximation were also derived for the real part of the self-energy of the DWP. Accounting for a nonlinear energy dependence of the barrier, leads to a slight asymmetry in the plus-minus quantities for the energy shift [Eqs. (29c), (30c)]. The treatment is general and concerns both the symmetric and the asymmetric DWP. When it is assumed that the degree of asymmetry is small, then the results [Eq. (42)] reduce to the ones obtained earlier by Miller.?

For the physics of the full potential, Fig. 1, which was the real object of this work, the complex poles of $G_{s c}(E)$ provide information about the tendency to a virtual equilibrium, oscillation and decay of the system, initially prepared in the left well. The related dynamics was examined using the relative sizes of the two barriers as a parameter. In this way, it is possible to observe the effect of an external perturbation, say an electric field.
In the follow up work, using the present results for the $G_{s c}(E)$ the semiclassical propagator will be computed, and hence the explicit time-dependence of this system will be examined.

${ }^{1}$ L. D. Landau and E. M. Lifshitz, Quantum Mechanics, $2^{\text {nd }}$ ed. (Pergamon, Oxford, 1965).

${ }^{2}$ E. Merzbacher, Quantum Mechanics, $3^{\text {rd }}$ ed. (Wiley, New York, 1998).

${ }^{3}$ J. Faist, F. Capasso, C. Sirtori, K. W. West, and L. N. Pfeiffer, Nature (London) 390, 589 (1997).

${ }^{4}$ Th. Mercouris and C. A. Nicolaides, J. Phys. B 24, L557 (1991).

${ }^{5}$ Th. G. Douvropoulos and C. A. Nicolaides, J. Phys. B 35, 4453 (2002).

${ }^{6}$ V. A. Benderskii, V. I. Goldanskii, and D. E. Makarov, Phys. Rep. 233, 195 (1993).

${ }^{7}$ W. H. Miller, J. Phys. Chem. 83, 960 (1979).

${ }^{8}$ B. R. Holstein, J. Phys. C 19, L279 (1986).

${ }^{9}$ B. R. Holstein, Am. J. Phys. 56, 338 (1988); ibid. 56, 894 (1988).

${ }^{10}$ R. P. Feynman and A. R. Hibbs, Quantum Mechanics and Path Integrals, (McGraw-Hill, New York, 1965).

${ }^{11}$ M. V. Berry and K. E. Mount, Rep. Prog. Phys. 35, 315 (1972).

${ }^{12}$ M. C. Gutzwiller, J. Math. Phys. 12, 343 (1971).

${ }^{13}$ D. W. McLaughlin, J. Math. Phys. 13, 1099 (1972).

${ }^{14}$ W. H. Miller, J. Chem. Phys. 56, 38 (1972); ibid., 63, 996 (1975); W. H. Miller and T. F. George, ibid. 56, 5668 (1972).

${ }^{15}$ B. R. Holstein and A. R. Swift, Am. J. Phys. 50, 829 (1982); ibid. 50, 833 (1982).

${ }^{16}$ B. R. Holstein, Am. J. Phys. 51, 897 (1983).

${ }^{17}$ A. M. Polyakov, Nucl. Phys. B 120, 429 (1977).

${ }^{18}$ S. Coleman, Aspects of Symmetry (Cambridge University Press, Cambridge, 1985).

${ }^{19}$ U. Weiss and W. Haeffner, Phys. Rev. D 27, 2916 (1983).

${ }^{20}$ G. V. Dunne, Found. Phys. 30, 463 (2000).

${ }^{21}$ M. I. Katsnelson, M. van Schilfgaarde, V. P. Antropov, and B. N. Harmon, Phys. Rev. A 54, 4802 (1996).

${ }^{22}$ N. Rom, N. Moiseyev, and R. Lefebvre, J. Chem. Phys. 95, 3562 (1991).

${ }^{23}$ Y. Guo, Y. Qin, D. C. Sorescu, and D. L. Thomson, J. Chem. Phys. 104, 4041 (1996).

${ }^{24}$ G. V. Mil'nikov, K. Yagi, T. Taketsugu, H. Nakamura, and K. Hirao, J. Chem. Phys. 119, 10 (2003).

${ }^{25}$ W. T. Strunz, J. Phys. A 25, 3855 (1992).

${ }^{26}$ F. M. Andrade, B. K. Cheng, M. W. Beims, and M. G. E. Da Luz, J. Phys. A 36, 227 (2003). 